\title{
Has the Indian lockdown averted deaths?
}

\author{
Suvrat Raju ${ }^{1}$ \\ International Centre for Theoretical Sciences, Tata Institute of Fundamental Research, Shivakote, \\ Bengaluru 560089, India. \\ E-mail: suvrat@icts.res.in
}

ABSTRACT: Within the context of SEIR models, we consider a lockdown that is both imposed and lifted at an early stage of an epidemic. We show that, in these models, although such a lockdown may delay deaths, it eventually does not avert a significant number of fatalities. Therefore, in these models, the efficacy of a lockdown cannot be gauged by simply comparing figures for the deaths at the end of the lockdown with the projected figure for deaths by the same date without the lockdown. We provide a simple but robust heuristic argument to explain why this conclusion should generalize to more elaborate compartmental models. We qualitatively discuss some conditions, beyond the scope of simple models, under which a lockdown might increase or decrease the epidemic's final toll in the real world. We conclude that SEIR models and their generalizations provide no reliable quantitative evidence that the Indian lockdown has averted any deaths from the COVID-19 pandemic.

\footnotetext{
${ }^{1}$ The views expressed in this article are personal and do not indicate the views of the author's institution.
} 


\section{Contents}

1 Introduction 1

2 Lockdowns in the SEIR model $\quad 2$

3 A heuristic explanation and general compartmental models $\quad 11$

4 Real-world caveats $\quad 14$

5 Conclusion and discussion $\quad 17$

$\begin{array}{ll}\text { A The INDSCI-SIM model } & 18\end{array}$

\section{Introduction}

India entered a nationwide lockdown on 24 March, in response to the COVID-19 pandemic. Although this lockdown still technically remains in force, as of the writing of this note, restrictions have progressively been eased. The lockdown has failed to prevent a rapid growth in the number of cases and deaths.

Nevertheless, on 22 May, the Indian government presented the results of numerous studies, all of which claimed that the lockdown had averted a significant number of deaths. One study by the Boston Consulting Group, which reportedly used a SEIR model, claimed that the lockdown had saved between 1,20,000 - 2,10,000 lives [1]. Subsequently, the modelling subgroup of the Indian Scientists Response to COVID-19 also announced that it had found, using its INDSCI-SIM model, that the lockdown had averted between 8,000 - 32,000 deaths by 15 May [2].

The purpose of this note is to explain why, even within the context of simple SEIR epidemiological models, and their generalizations, such claims are misleading. This is because such models uniformly predict that lockdowns that are implemented early in the epidemicand fail to quash the epidemic - have a negligible impact on the final death toll. While a lockdown may delay deaths, the models themselves suggests that the fatalities in a "lockdown scenario" will rapidly catch up with fatalities in a "no lockdown scenario."

Therefore these models teach us that the comparison of fatalities between two scenarios by an arbitrarily chosen fixed date - in this case, 15 May - is an absurd metric to gauge the efficacy of a lockdown. This conclusion is described in section 2 .

Epidemiological models are known to be often unreliable, and their predictions are sensitive to the parameters one chooses. However, the specific property of the models described

above is very robust and insensitive to the parameters chosen. Moreover, while we use a 
SEIR model to obtain specific analytic results, we explain in section 3 that our results should qualitatively generalize to more complicated models. Of course, one may choose to eschew models altogether. But to the extent that we rely on epidemiological models at all, one of the few reliable lessons we should take away is that lockdowns should not be evaluated by comparing fatalities by a fixed date.

In the real world, lockdowns may have a positive or negative impact on the epidemic that may be difficult to capture in the model. For instance, if the lockdown quashes the epidemic, or can be used to greatly ramp up healthcare capacity, it may reduce the final toll of the epidemic significantly. On the other hand, if the lockdown engenders economic insecurity, and makes it harder for people to implement long-term precautions like physical distancing, then it may have a negative impact on the eventual toll of the epidemic. We list these effects qualitatively in section 4 . Nevertheless, we explain how these effects are much more important for the final toll of the epidemic than the direct effect of the lockdown itself. In the Appendix we discuss the INDSCI-SIM model, as an example of an extended SEIR model that confirms our general expectations.

The conclusion of this note is that simple epidemiological models are unable to provide any reliable quantitative evidence that the lockdown in India has averted deaths. Rather, an analysis of whether the lockdown has been successful or not must rely on balancing the qualitative factors described in 4 . It seems apparent to us that a consideration of these factors suggests that the Indian lockdown has contributed to worsening the final toll of the epidemic.

We would like to state two caveats at the outset

1. The results presented here involve an elementary analysis of epidemiological models, and are well understood by public health workers and most epidemiologists. We feel the need re-emphasize these elementary results, only in light of the claims mentioned above.

2. At various points in this analysis, we use assumptions that are appropriate for the lockdown in India but may not apply to other countries. So the analysis here should not be construed as a comment on lockdowns in other parts of the world.

\section{Lockdowns in the SEIR model}

We start by considering the simplest epidemiological models - the SEIR models. It is possible to obtain simple analytic results in these models. A SEIR model was reportedly also used in the BCG study, and the INDSCI-SIM model can also be thought of as an extended SEIR model.

However, we will explain in section 3 why the essential elements of our discussion are robust and model independent, and why we expect them to generalize to other models as well. 


\subsection{Setting}

The SEIR model starts by dividing the population into four different compartments. The population is divided into a susceptible group (S), an exposed group (E), an infected group (I) and a removed group (R), which includes those who have recovered as well as those deceased. The differential equations that govern the model are very simple [3].

$$
\begin{array}{ll}
\frac{d S}{d t}=\frac{-\beta_{1} S I}{N} ; & \frac{d E}{d t}=\frac{\beta_{1} S I}{N}-\beta_{2} E ; \\
\frac{d I}{d t}=\beta_{2} E-\beta_{3} I ; & \frac{d R}{d t}=\beta_{3} I .
\end{array}
$$

We ignore natural births and deaths since they are not relevant for this analysis.

All the variables above are functions of time. But, as above, instead of writing $S(t)$, we will generally suppress this dependence by writing $S$. We only display the time-dependence if we wish to refer to a specific time. For instance, $S(0)$ is the number of susceptible individuals at time 0 , taken to be the start of the epidemic, and similar conventions hold for the other variables.

Here, $N$ is the total population size at the start of the epidemic. We assume that $S(0)+E(0)+I(0)+R(0)=N$. This is clearly conserved by the equations.

In analyzing the model, it is convenient to define a variable $F=I+E$, which satisfies

$$
\frac{d F}{d t}=I\left(\beta_{1} \frac{S}{N}-\beta_{3}\right)
$$

Moreover, $S$ is itself a monotonically decreasing function in this model, which is clear from the first equation above. It is useful, especially for the analysis of asymptotic properties, to reparameterize the time-variable in terms of the instantaneous value of the susceptible population. This leads to

$$
\frac{d F}{d S}=\left(\frac{\beta_{3} N}{\beta_{1} S}\right)-1
$$

This can be easily integrated to yield the path of the epidemic in $S-F$ space, which is

$$
\frac{S}{N}-\frac{\beta_{3}}{\beta_{1}} \log \left(\frac{S}{N}\right)+\frac{F}{N}=\mathcal{K}
$$

Here we have introduced a new constant $\mathcal{K}$, which identifies the trajectory of the epidemic.

We may set $\mathcal{K}$ using the initial conditions. For instance, if we assume that the epidemic is seeded by a single exposed case, $F(0)=1$ and that the entire population, except for this individual, is initially susceptible: $S(0)=N-1$. Then we find that $\mathcal{K}=1-\frac{\beta_{3}}{\beta_{1}} \log \left(1-\frac{1}{N}\right)$ for the initial conditions above. Since, for a large population, $\frac{1}{N} \ll 1$, we may simply approximate $\mathcal{K} \approx 1$ for this set of initial conditions. But equation (2.4) is valid for other initial conditions as well.

The constant $\mathcal{K}$ is important because it allows us to compute the size of the epidemic as follows. At late times, the epidemic ends and so $F(\infty)=0$. Therefore the final susceptible 
fraction, $s_{\infty}=S(\infty) / N$ is just given by solving

$$
s_{\infty}-\frac{\beta_{3}}{\beta_{1}} \log s_{\infty}=\mathcal{K} .
$$

We will be interested in the case where $\beta_{1}>\beta_{3}$, which is when the epidemic grows exponentially at the start. Then (2.5) has a single root in the range $s_{\infty} \in\left(0, \frac{S(0)}{N}\right)$, which is the one we are interested in.

To compute fatalities, we assume a fatality rate, $\mu$. This means that a fraction $\mu$ of all those infected do not recover but instead succumb to the infection. Note that this number is still counted in the variable $R$ and the fatalities, at any point of time, are just $\mu R$. Then the final number of fatalities, which we denote by $D_{\infty}$, is given by

$$
D_{\infty}=\mu N\left(1-s_{\infty}\right)
$$

where $s_{\infty}$ solves the equation (2.5) above.

We will compare multiple scenarios below. The parameter that we vary in these scenarios is $\beta_{1}$ and the quantity we examine is $s_{\infty}$. To avoid confusion, we adopt the convention that whenever these variables appear without a subscript, eg. $\beta_{1}$ or $s_{\infty}$, they refer to the generic variable and not a particular value. On the other hand, expressions like $\beta_{1}^{(a)}$ or $s_{\infty}^{(l)}$ refer to the values of these variables in a specific scenario.

\subsection{Definition of scenarios}

In this note, we are concerned with the effect of a lockdown both imposed and lifted early in the epidemic. We make these terms precise below and compare this scenario with a nolockdown scenario.

\section{Lockdown scenario}

In the lockdown scenario, we consider an epidemic that starts with some initial value of $F(0)$ and $S(0)=N-F(0)$. The epidemic starts to evolve freely with an initial value of $\beta_{1}=\beta_{1}^{(b)}$. At $1-\frac{S}{N}=\epsilon_{1} \ll 1$, the value of $\beta_{1}$ is abruptly changed to $\beta_{1}^{(l)}$ where $\beta_{1}^{(l)}<\beta_{1}^{(b)}$. Then the epidemic evolves with these new parameters until $1-\frac{S}{N}=\epsilon_{2} \ll 1$. Note that we always have $\epsilon_{2}>\epsilon_{1}$. Then $\beta_{1}$ is changed again to $\beta_{1}^{(a)}$ such that $\beta_{1}^{(l)}<\beta_{1}^{(a)}<\beta_{1}^{(b)}$, and then the epidemic evolves freely till its end.

The interpretation of the scenario above should be obvious. At the beginning, the epidemic evolves with some initial parameters. A lockdown enforces physical distancing and brings down the contact rate between individuals. It does not change the biological parameters of the infection, including its incubation period or time to recovery, and therefore it is modelled by a decrease in $\beta_{1}$. When the lockdown ends, the contact rate does not necessarily return to its initial value. Since several measures, including some measure of physical distancing and perhaps behavioural alterations and other precautions (such as mask-wearing) remain in place after the lockdown, the model allows for a third value of $\beta_{1}$ in the post-lockdown 
phase. This third value is expected to be in-between the pre-lockdown value, and the value during the lockdown.

The fact that the lockdown is imposed early and ends early during the epidemic is indicated by $\epsilon_{1} \ll 1$ and $\epsilon_{2} \ll 1$.

\section{No-lockdown scenario}

In the no-lockdown scenario, we consider an epidemic that starts with the same value of $F(0)$ as above and again take $S(0)=N-F(0)$. The epidemic again initially evolves with $\beta_{1}=\beta_{1}^{(b)}$. For $1-\frac{S}{N} \in\left(\epsilon_{1}, \epsilon_{2}\right)$, we take $\beta_{1}$ to vary as some monotonic function $\beta_{1}\left(1-\frac{S}{N}\right)$ with the condition that $\beta_{1}\left(\epsilon_{1}\right)=\beta_{1}^{(b)}$ and $\beta_{1}\left(\epsilon_{2}\right)=\beta_{1}^{(a)}$. The details of this monotonic function will be unimportant for our purpose. Beyond $1-\frac{S}{N}=\epsilon_{2}$, the epidemic evolves with $\beta_{1}=\beta_{1}^{(a)}$ until its end.

The interpretation of this scenario should also be obvious. At the beginning of the epidemic, it evolves with some initial parameters. At some point, behavioural changes are introduced through physical distancing and other precautions. These are the same changes and precautions that set $\beta_{1}=\beta_{1}^{(a)}$ in the lockdown scenario. These changes can often be implemented rapidly and, in fact, they can be implemented instead of the lockdown. So it would be quite reasonable to approximate the function $\beta_{1}\left(1-\frac{S}{N}\right)$ by a step function with a term proportional to $\theta\left(1-\frac{S}{N}-\epsilon_{1}\right)$. Nevertheless, we consider a more general scenario where these behavioural changes and precautions are implemented more gradually. But we do assume that they are in place by the time the epidemic has reached the stage, $1-\frac{S}{N}=\epsilon_{2}$, which is the stage of susceptibility where the lockdown ends in the "lockdown scenario".

However, note, in particular, that the rate, $\beta_{1}$, in the lockdown scenario is always lower or equal to its value in the no-lockdown scenario.

\subsection{Effect of a lockdown on fatalities}

We now compare final fatalities in the lockdown and no-lockdown scenarios.

\section{Fatalities in the lockdown scenario}

The change in parameters, upon the imposition of the lockdown, also leads to a change in the value of $\mathcal{K}$ by $\delta \mathcal{K}_{11}$, which is given by

$$
\delta \mathcal{K}_{11}=\left(\frac{1}{\beta_{1}^{(b)}}-\frac{1}{\beta_{1}^{(l)}}\right) \beta_{3} \log \left(1-\epsilon_{1}\right) .
$$

This equation is derived by noting that $S$ and $F$ remain continuous when the lockdown is imposed, and the change in $\mathcal{K}$ comes only from the change in the value of $\beta_{1}$. When the lockdown is lifted, there is an additional change in this constant, $\delta \mathcal{K}_{12}$, which is given by

$$
\delta \mathcal{K}_{12}=\left(\frac{1}{\beta_{1}^{(l)}}-\frac{1}{\beta_{1}^{(a)}}\right) \beta_{3} \log \left(1-\epsilon_{2}\right) .
$$


The net change in $\mathcal{K}$ can be expanded out to first order in $\epsilon_{1}$ and $\epsilon_{2}$ as

$$
\delta \mathcal{K}_{1}=\delta \mathcal{K}_{11}+\delta \mathcal{K}_{12}=-\beta_{3}\left(\frac{\epsilon_{2}-\epsilon_{1}}{\beta_{1}^{(l)}}+\frac{\epsilon_{1}}{\beta_{1}^{(b)}}-\frac{\epsilon_{2}}{\beta_{1}^{(a)}}\right)+\mathcal{S}_{1},
$$

where we have clubbed the higher order terms into $\mathcal{S}_{1}$, since they will be largely irrelevant for our analysis.

$$
\mathcal{S}_{1}=-\frac{\beta_{3}\left(\epsilon_{1}^{2} \beta_{1}^{(a)}\left(\beta_{1}^{(l)}-\beta_{1}^{(b)}\right)+\beta_{1}^{(b)} \epsilon_{2}^{2}\left(\beta_{1}^{(a)}-\beta_{1}^{(l)}\right)\right)}{2 \beta_{1}^{(l)} \beta_{1}^{(b)} \beta_{1}^{(a)}}+O\left(\epsilon^{3}\right) .
$$

It is convenient to compare the final toll of the epidemic by comparing it with a "reference" epidemic, where the value of $\beta_{1}=\beta_{1}^{(a)}$ throughout the epidemic, and where the epidemic starts with the same initial value of $\mathcal{K}$ as the epidemic above. If we define the solution of (2.5) for this reference case as $s_{\infty}^{\text {ref }}$ and if we denote the value of $s_{\infty}$ in the lockdown scenario by $s_{\infty}^{(l)}$, then we can then use equation (2.5) to compute the difference between these two quantities.

To first order, this is controlled by

$$
\frac{d s_{\infty}}{d \mathcal{K}}=-\left(\frac{\beta_{3}}{\beta_{1}^{(a)} s_{\infty}}-1\right)^{-1}
$$

where the quantity in brackets is positive. And therefore

$$
s_{\infty}^{(l)}-s_{\infty}^{\mathrm{ref}}=\left(\frac{\beta_{3}}{\beta_{1}^{(a)} s_{\infty}^{\mathrm{ref}}}-1\right)^{-1} \beta_{3}\left(\frac{\epsilon_{2}-\epsilon_{1}}{\beta_{1}^{(l)}}+\frac{\epsilon_{1}}{\beta_{1}^{(b)}}-\frac{\epsilon_{2}}{\beta_{1}^{(a)}}\right)+\mathrm{O}\left(\epsilon^{2}\right) .
$$

\section{Fatalities in the no-lockdown scenario}

In the "no lockdown" scenario, we have an unknown function $\beta_{1}\left(1-\frac{S}{N}\right)$ which controls how fast the long-term equilibrium value of $\beta_{1}=\beta_{1}^{(a)}$ is achieved. Even though this function is unknown, under the assumption that it is monotonic, we can bound the asymptotic value of $s_{\infty}$ both above and below by considering two extreme subcases within this scenario.

First consider the case where the value of $\beta_{1}$ changes abruptly to $\beta_{1}^{(a)}$ when $1-\frac{S}{N}=\epsilon_{1}$. In this case, the epidemic shifts to a new trajectory where $\mathcal{K}$ changes by

$$
\delta \mathcal{K}_{21}=\beta_{3} \log \left(1-\epsilon_{1}\right)\left(\frac{1}{\beta_{1}^{(b)}}-\frac{1}{\beta_{1}^{(a)}}\right)=-\beta_{3} \epsilon_{1}\left(\frac{1}{\beta_{1}^{(b)}}-\frac{1}{\beta_{1}^{(a)}}\right)+\mathrm{O}\left(\epsilon^{2}\right) .
$$

The second sub-scenario is the case where the value of $\beta_{1}$ stays constant until $1-\frac{S}{N}=\epsilon_{2}$ and then changes abruptly to $\beta_{1}^{(a)}$. In this case, the value of $\mathcal{K}$ changes by

$$
\delta \mathcal{K}_{22}=\beta_{3} \log \left(1-\epsilon_{2}\right)\left(\frac{1}{\beta_{1}^{(b)}}-\frac{1}{\beta_{1}^{(a)}}\right)=-\beta_{3} \epsilon_{2}\left(\frac{1}{\beta_{1}^{(b)}}-\frac{1}{\beta_{1}^{(a)}}\right)+\mathrm{O}\left(\epsilon^{2}\right) .
$$

The reason to consider these two sub-scenarios is that the final value of $s_{\infty}$ in the "no lockdown" scenario is bounded on both sides by its value in the cases above. In particular, 
denote the final value of the susceptibility in an arbitrary no-lockdown scenario (as specified above) by $s_{\infty}^{(n)}$. Then, we find that independent of the details of the variation of $\beta_{1}\left(1-\frac{S}{N}\right)$ in the range $1-\frac{S}{N} \in\left(\epsilon_{1}, \epsilon_{2}\right)$ in this scenario, we have

$$
s_{\infty}^{\mathrm{ref}}-s_{\infty}^{(n)} \geq s_{\infty}^{\mathrm{ref}}-s_{\infty}^{\left(n_{1}\right)}=\left(\frac{\beta_{3}}{\beta_{1}^{(a)} s_{\infty}^{\mathrm{ref}}}-1\right)^{-1} \beta_{3} \epsilon_{1}\left(\frac{1}{\beta_{1}^{(a)}}-\frac{1}{\beta_{1}^{(b)}}\right) .
$$

We also have

$$
s_{\infty}^{\mathrm{ref}}-s_{\infty}^{(n)} \leq s_{\infty}^{\mathrm{ref}}-s_{\infty}^{\left(n_{2}\right)}=\left(\frac{\beta_{3}}{\beta_{1}^{(a)} s_{\infty}^{\mathrm{ref}}}-1\right)^{-1} \beta_{3} \epsilon_{2}\left(\frac{1}{\beta_{1}^{(a)}}-\frac{1}{\beta_{1}^{(b)}}\right) .
$$

Here $s_{\infty}^{\left(n_{1}\right)}$ and $s_{\infty}^{\left(n_{2}\right)}$ are the final susceptible fractions in the two extreme no-lockdown scenarios.

\section{Difference in fatalities}

The difference in fatalities in the two scenarios is proportional to this difference in $s_{\infty}$ in the two scenarios. We have

$$
\delta D=\mu N\left(s_{\infty}^{(l)}-s_{\infty}^{(n)}\right) .
$$

Even though we have an unknown interpolating function in the no-lockdown scenario we can bound $\delta D$ both from above and below. We find that the factors conspire to give the simple result

$$
\frac{\mu N \beta_{3}}{\left(\frac{\beta_{3}}{\beta_{1}^{(a)} s_{\infty}^{\mathrm{ref}}}-1\right)}\left(\epsilon_{2}-\epsilon_{1}\right)\left(\frac{1}{\beta_{1}^{(l)}}-\frac{1}{\beta_{1}^{(a)}}\right) \leq \delta D \leq \frac{\mu N \beta_{3}}{\left(\frac{\beta_{3}}{\beta_{1}^{(a)} s_{\infty}^{\mathrm{ref}}}-1\right)}\left(\epsilon_{2}-\epsilon_{1}\right)\left(\frac{1}{\beta_{1}^{(l)}}-\frac{1}{\beta_{1}^{(b)}}\right) .
$$

We will use the notation $(\delta D)^{\left(n_{1}\right)}$ for the lower bound above and $(\delta D)^{\left(n_{2}\right)}$ for the upper bound. These correspond to the difference in deaths between the lockdown scenario and the two extreme no-lockdown scenarios detailed above.

The reason that this effect is small is because it is proportional to $\epsilon_{1}$ and $\epsilon_{2}$ and, by assumption, these numbers are small for the "early lockdown" scenario that we consider.

\section{Deaths averted and deaths during lockdown}

In fact, a useful estimate of the figure of deaths averted is given by the number of deaths that take place during the lockdown.

Note that the fatalities observed during the lockdown can be computed from (2.4) to be

$$
\delta D^{(l)}=\mu N \frac{\beta_{3}}{\beta_{1}^{(l)}} \delta(\log (S)) \approx \mu N \frac{\beta_{3}}{\beta_{1}^{(l)}}\left(\epsilon_{2}-\epsilon_{1}\right)+\mathrm{O}\left(\epsilon^{2}\right) .
$$

In particular we see that

$$
\frac{1-\frac{\beta_{1}^{(l)}}{\beta_{1}^{(a)}}}{\left(\frac{\beta_{3}}{\beta_{1}^{(a)} s_{\infty}^{\mathrm{ref}}}-1\right)} \leq \frac{\delta D}{\delta D^{(l)}} \leq \frac{1-\frac{\beta_{1}^{(l)}}{\beta_{1}^{(b)}}}{\left(\frac{\beta_{3}}{\beta_{1}^{(a)} s_{\infty}^{\mathrm{ref}}}-1\right)}
$$


The $\mathrm{O}(1)$ factors that appear above depend, in detail, on the various constants in the problem. In fact, for several reasonable values of the parameters, these factors are smaller than 1 meaning that deaths averted are smaller than the deaths that have taken place during the lockdown. But, in any case, an immediate implication is that one should be skeptical of estimates that suggest that deaths averted are much higher than deaths during the lockdown. In India's case, since 2743 deaths had been recorded during the lockdown, from 24 March to 15 May [4], this immediately tells us that the significantly higher figures described in the Introduction are absurd, even within the context of epidemiological models.

Here, we would like emphasize an important general point. In the SEIR model, the figure for deaths averted by the lockdown is entirely separate from the figure for the deaths that take place in the same time period in the no-lockdown scenarios. If one considers an "effective lockdown" that is imposed early, then even after a long time (in units set by $\beta_{1}^{-1}, \beta_{2}^{-1}, \beta_{3}^{-1}$ ) the value of $\epsilon_{2}$ may remain very small. In the meantime, the deaths in the no-lockdown scenario in the same time period will diverge exponentially from the deaths in the lockdown scenario. It is this exponentially large difference that gives rise to the figures described in the Introduction. But, within the model, this large number is irrelevant for the final figure of the deaths averted.

\subsection{A numerical example}

The results above might seem puzzling, since "common sense" suggests that a lockdown should "avert deaths". Before we explain them heuristically, we provide a simple numerical example.

The point of this example is not to make realistic predictions for the Indian epidemic. The model is too simple to do that, and moreover the available data-including that of the total number of cases and fatalities - is too flawed. Our point is only to illustrate the theoretical effect described above. For this reason, we deliberately do not consider the fatality data for India.

Instead we consider a hypothetical epidemic in a population of $N=10^{7}$. The epidemic is seeded by a small set of exposed cases: $E(0)=10, I(0)=0, S(0)=N-10$. The fatality rate is taken to be $\mu=.005$ or $0.5 \%$. We assume that $\beta_{2}=\frac{1}{3}$ (day) ${ }^{-1}$ and $\beta_{3}=\frac{1}{5}$ (day $)^{-1}$. In our hypothetical example, before the lockdown, the epidemic grows rapidly with $\beta_{1}^{(b)}=\frac{3}{5}(\text { day })^{-1}$. In the lockdown scenario, a lockdown of 70 days is imposed on day 20 of the epidemic and lifted on day 90 of the epidemic. The lockdown brings $\beta_{1}$ down to $\beta_{1}^{(l)}=\frac{3}{10}(\text { day })^{-1}$. This lockdown corresponds to the values of $\epsilon_{1}=4.8 \times 10^{-5}$ and $\epsilon_{2}=4.9 \times 10^{-3}$ and therefore our assumption of an "early lockdown" is met. At the end of the lockdown, $\beta_{1}$ rises again

to $\beta_{1}^{(a)}=\frac{2}{5}$ (day $)^{-1}$. It does not rise to its original value, since some sustainable behavioural changes are made and some long-term precautions are put in place.

We compare this lockdown scenario with the two "no lockdown" scenarios described above. In one scenario, denoted by "NL1" in the graphs below, the long-term precautions changes are implemented, instead of the lockdown on day 20. In the other scenario, denoted by "NL2", the epidemic continues to evolve freely past day 20 . The long-term precautions 
are implemented only when $1-\frac{S}{N}=\epsilon_{2}$. In terms of time, this value of $\epsilon_{2}$ is reached at 44.90 days. Therefore, in the scenario NL2, the long-term precautions and behavioural changes that bring $\beta_{1}$ down from $\beta_{1}^{(b)}$ to $\beta_{1}^{(a)}$ are put in place 25 days after the lockdown is imposed in scenario "L". As explained above, if we take a more general variation of $\beta_{1}$ we expect the asymptotic fatalities to be bounded above and below by these two scenarios.

The fatalities as a function of time in the three scenarios are shown in Figure 1. This

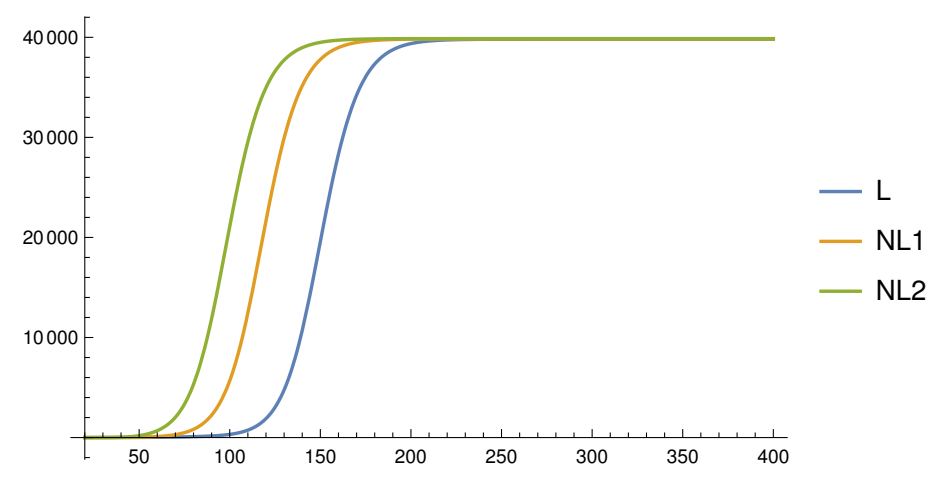

Figure 1: Total deaths as a function of time in the three scenarios. "L" is the lockdown followed by long-term precautions; NL1 implements only the long-term precautions instead of the lockdown; NL2 implements long-term precautions after a delay.

figure perfectly bears out the analysis above. When one compares the lockdown scenario to NL2 scenario on day 90, the no-lockdown scenario has had 11,722 excess deaths. However, it is absurd to conclude from this figure that the lockdown has "averted more than 11,000 deaths". This is because, at the end of 365 days, deaths in the lockdown scenario have caught up. In fact, in the model, there are less than 56 excess deaths after 365 days compared to the lockdown scenario.

The comparison with the NL1 scenario is similar. Here, in the model, there are 2078 excess deaths compared to the lockdown scenario by day 90 . But after 365 days, the difference in deaths drops below 28 .

The difference in deaths between the lockdown scenario and the NL1 and NL2 scenarios is also plotted in Figure 2. This graph shows that the difference in deaths appears to become very large at intermediate times, but then drops to a very small number by the end of the epidemic.

The example also provides us with an opportunity to check our first order analytical formulas. The match between the numerics and the first order approximations is very good 


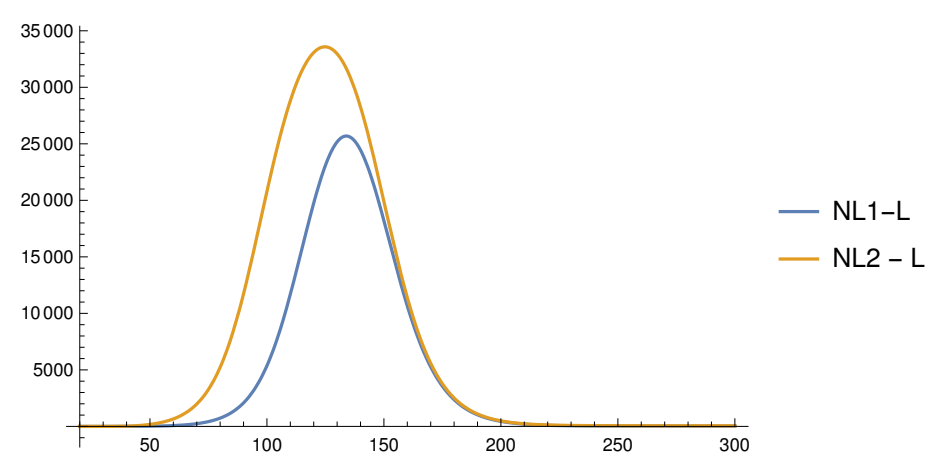

Figure 2: Death delayed by the lockdown. The graph shows the difference in fatalities between the lockdown scenario and the no-lockdown scenarios as a function of time. By the end of the epidemic, deaths in the lockdown scenario catch up almost entirely with the no-lockdown scenarios.

as shown in the table below.

\begin{tabular}{|c|c|c|c|}
\hline Quantity & Numerical $(N)$ & First-order $\operatorname{Approx}(A)$ & Error $\left(\frac{|N-A|}{N}\right)$ \\
\hline$\delta \mathcal{K}_{1}$ & $-8.06 \times 10^{-4}$ & $-8.04 \times 10^{-4}$ & $2.5 \times 10^{-3}$ \\
$\delta \mathcal{K}_{21}$ & $8.03 \times 10^{-6}$ & $8.03 \times 10^{-6}$ & $2.4 \times 10^{-5}$ \\
$\delta \mathcal{K}_{22}$ & $8.22 \times 10^{-4}$ & $8.20 \times 10^{-4}$ & $2.5 \times 10^{-3}$ \\
$\delta D^{(l)}$ & 27.64 & 27.51 & $4.8 \times 10^{-3}$ \\
$\delta D^{\left(n_{1}\right)}$ & 27.92 & 27.78 & $4.7 \times 10^{-3}$ \\
$\delta D^{\left(n_{2}\right)}$ & 55.70 & 55.57 & $2.4 \times 10^{-3}$ \\
\hline
\end{tabular}

We also note that for these parameters, the lower and upper bounds that appear in (2.19) are 0.17 and 0.34 respectively. In this model, the deaths that take place during the lockdown are given by $\delta D^{(l)}=162.75$. As expected, the two extreme no-lockdown solutions saturate the lower and upper bound of (2.19). So, in this example, the deaths averted are significantly smaller than the deaths that take place during the lockdown.

The reader might wonder, from Figure 1 whether, in the lockdown scenario, the peak of infections is "flattened". However, even this does not happen. For the parameters above, although the peaks occur at significantly different times, the actual peak values of $I$ in the NL2, NL1 and L scenarios are similar and given by

$$
I_{\max }^{\left(n_{2}\right)}=9.56 \times 10^{5} ; \quad I_{\max }^{\left(n_{1}\right)}=9.51 \times 10^{5} ; \quad I_{\max }^{(l)}=9.46 \times 10^{5} .
$$

So, we have

$$
\frac{I_{\max }^{\left(n_{2}\right)}-I_{\max }^{(l)}}{N}=1.0 \times 10^{-3} ; \quad \frac{I_{\max }^{\left(n_{1}\right)}-I_{\max }^{(l)}}{N}=5.0 \times 10^{-4} .
$$

This is entirely consistent with the understanding that we develop in section 3. Although the focus of this note is on "deaths averted" in fact, in reasonable models, all the intrinsic 
properties of the trajectory with an early lockdown are very close to those of the trajectory without the lockdown.

\section{A heuristic explanation and general compartmental models}

Epidemiological models are often unreliable and subject to sensitivity in the parameters. In fact, this author is of the opinion that the only predictions of models that should be regarded as robust and taken seriously are those that can also be reproduced by a simple heuristic argument. So in this section we provide a heuristic argument to explain the result above, which states that a lockdown has only a small impact on the final number of deaths. We then use our heuristic explanation to generalize our result to extensions of the SEIR model.

\subsection{The SEIR Model}

The formula (2.17) has two aspects. The first aspect, which is the important one qualitatively, is that the result for deaths averted is $\mathrm{O}(\epsilon)$. This means that, in the limit, where we take the lockdown to be imposed and lifted earlier and earlier in the epidemic, its effect on the final number of fatalities vanishes. This is easy to understand. In such a situation, the lockdown only translates the entire curve of infections to the right, without having any appreciable impact on its shape. In particular, at leading order, an early lockdown does not avert fatalities at all.

This is a robust argument, and independent of the details of the model. Therefore we expect that, even in more complicated models, lockdowns, by themselves, should be ineffective in reducing deaths.

The formula (2.17) also has an $\mathrm{O}(\epsilon)$ piece and this term can also be understood through a slightly more elaborate heuristic argument. The key point is to track the epidemic's progress in terms of the fraction of the population that remains susceptible, rather than directly use the time-variable. Even in more complicated models, with a number of compartments, as long as recovery from infection grants immunity, the fraction of the population that remains susceptible is a monotonic function and so it can be used to track the progress of the epidemic.

Let us use this to understand how the results obtained above for the SEIR model can be understood heuristically. In this model, at the start when $1-\frac{S}{N} \approx 0$, each individual effectively infects $\frac{\beta_{1}^{(b)}}{\beta_{3}}$ other individuals. In one of the extreme no-lockdown scenarios above, when $1-\frac{S}{N}=\epsilon_{2}$, this number is lowered to $\frac{\beta_{1}^{(a)}}{\beta_{3}}$. The epidemic grows until $\frac{S}{N}=\frac{\beta_{3}}{\beta_{1}^{(a)}}$. Beyond this threshold, the epidemic starts to decay but it does not end. Importantly the final toll of the epidemic is controlled by the threshold where it starts to decay but also the number of exposed and infected individuals at this threshold. These individuals cause an "overshoot" beyond the "herd immunity" threshold and increase the final toll of the epidemic.

Now the lockdown alters this trajectory as follows. First note that since it takes longer for the population to reach the level $1-\frac{S}{N}=\epsilon_{2}$, the lockdown tends to advance recoveries over the no-lockdown scenario. This effect is clearly proportional to $\epsilon_{2}-\epsilon_{1}$. But this implies that 
the total number of infected and exposed individuals are smaller in the lockdown scenario than in the no-lockdown scenario, when the two epidemics are compared at the same value of $1-\frac{S}{N}$. This effect is proportional to $\left(\epsilon_{2}-\epsilon_{1}\right)$ since $R+F+S=N$ and so the change in $F$ is precisely the change in $S$. This effect causes the epidemic to end at a lower value of $\frac{S}{N}$ than without the lockdown. Since the final number of fatalities is controlled by the end-point of the epidemic, one sees that the lockdown lowers fatalities by a term that is proportional to $\epsilon_{2}-\epsilon_{1}$.

The comparison of the lockdown scenario with the other extreme no-lockdown scenario - where behavioural changes are implemented instead of the lockdown and at the same time as the lockdown - is similar. One again finds that fatalities in the lockdown scenario are lower by a factor that is proportional to $\epsilon_{2}-\epsilon_{1}$.

\subsection{General compartmental models}

We now explain why we expect the heuristic argument above to continue to apply to more general compartmental models.

In more complicated models, the population is often divided into additional compartments. For instance, instead of simply considering "infected individuals" one may divide them into a group that is "asymptomatic" and another that is "symptomatic". The details of these compartments are not important for our argument and say that we have divided the population into $Q$-compartments, whose occupancy we denote by $\mathcal{C}_{i}$, with $i=1 \ldots Q$.

The dynamics associated with the additional compartments will also be different from the SEIR model. For instance, "asymptomatic" and "symptomatic" individuals might pass on the infection to susceptible individuals at different rates. The details of these dynamics are also not important for the argument, and say that the trajectory of the epidemic without the lockdown follows a path

$$
\frac{d \mathcal{C}_{i}^{(n)}}{d S}=\mathcal{F}_{i}\left(\frac{S}{N}, \frac{\mathcal{C}_{i}^{(n)}}{N}\right)
$$

for some set of functions $\mathcal{F}_{i}$. These functions are $\mathrm{O}(1)$ functions of $\mathrm{O}(1)$ quantities and not expected to scale with $N$ since the left hand side does not scale with $N$ either. This represents the fact that in such models, the important quantity is the fraction of the total population that is in a compartment and not the absolute number for the occupancy of that compartment.

The framework described by (3.1) is quite general. For instance, it can be used to easily model a fatality rate that is a function of the instantaneous number of infections or the occupancy of another compartment in the model. To account for this possible effect, we introduce a compartment for fatalities, which we call $\mathcal{C}_{D}$. In the SEIR model, with a constant death-rate, this would always just be proportional to recoveries, but if the fatality rate depends on other variables, it might be different.

Note also that, in writing the equations in the form (3.1), where the trajectory is parameterized by the susceptibility, we have assumed that there is no explicit time-dependence in the parameters that govern the model. This assumption may be false if, for instance, the 
healthcare system undergoes a rapid improvement over a short period of time. We return to this issue in section 4 .

Now, a lockdown alters the dynamics of the epidemic for a limited period. This means that the lockdown changes the trajectory of the epidemic to one that satisfies

$$
\frac{d \mathcal{C}_{i}^{(l)}}{d S}=\mathcal{F}_{i}\left(\frac{S}{N}, \frac{\mathcal{C}_{i}^{(l)}}{N}\right)+\theta\left(1-\frac{S}{N}-\epsilon_{1}\right) \theta\left(\epsilon_{2}-1+\frac{S}{N}\right) \mathcal{G}_{i}\left(\frac{S}{N}, \frac{\mathcal{C}_{i}^{(l)}}{N}\right) .
$$

Here, during the lockdown, which starts when the fraction of the susceptible population is $1-\epsilon_{1}$ and lasts till it becomes $1-\epsilon_{2}$, we have assumed that the trajectory is controlled by some new evolution functions that differ from the old ones by $\mathcal{G}_{i}$.

One may choose to set the start and end-points of the lockdown in terms of time, rather than susceptibility, but equation (3.2) still applies with $\epsilon_{1}$ and $\epsilon_{2}$ merely being set by the value of $1-\frac{S}{N}$ at the starting and ending points of the lockdown.

Since the impact of the lockdown is constrained to the interval $1-\frac{S}{N} \in\left(\epsilon_{1}, \epsilon_{2}\right)$, the trajectories (3.1) and (3.2) satisfy the same differential equation both before and after this interval. In particular, if we compare the trajectories of the two scenarios after the lockdown, then it is only the initial conditions for these two trajectories that are different. Moreover, these initial conditions differ by only a small amount. This can be written as

$$
\frac{1}{N}\left|\mathcal{C}_{i}^{(l)}\left(S=N\left(1-\epsilon_{2}\right)\right)-\mathcal{C}_{i}^{(n)}\left(S=N\left(1-\epsilon_{2}\right)\right)\right|=\left(\epsilon_{2}-\epsilon_{1}\right) \rho_{i},
$$

where $\rho_{i}$ is some set of model-dependent $\mathrm{O}(1)$ constants controlled by the manner in which the lockdown modifies dynamics.

In the SEIR model the "termination condition" for the epidemic was simply $F=0$. Say that this termination condition generalizes to

$$
\mathcal{T}\left(S, \mathcal{C}_{i}\right)=0
$$

in the more elaborate model. We are interested in the value of $S$, at which (3.4) is satisfied and the expected fatalities can then be computed as $\mathcal{C}_{D}(S)$. But as a result of (3.3), we expect that if $(3.4)$ is solved by some value $S^{(l)}$ for the lockdown trajectory and some other value $S^{(n)}$ for the no-lockdown trajectory then

$$
\frac{\mathcal{C}_{D}\left(S^{(n)}\right)}{N}-\frac{\mathcal{C}_{D}\left(S^{(l)}\right)}{N}=\left(\epsilon_{2}-\epsilon_{1}\right) \sigma
$$

where $\sigma$ is again some $\mathrm{O}(1)$ constant that depends on the model.

The assumption here is that the differential equations that govern the epidemic, (3.1), are not chaotic so that a small difference in the initial conditions, (3.3), leads to a small difference in the asymptotic value of $\frac{S}{N}$. This is a reasonable assumption to make about epidemiological models. $^{1}$

\footnotetext{
${ }^{1}$ As discussed in section 4 there are some exceptions to this assumption. For instance, it may happen that when the $\mathcal{C}_{i}$ are below some threshold, the healthcare system can keep them below that threshold indefinitely and not otherwise. In such a case, small differences in initial conditions could make a significant difference in the final outcome. These exceptions, which apply when the epidemic is quashed by the lockdown, do not apply to the Indian lockdown as explained in section 4.
} 
To summarize: while the precise factors in (2.17) are specific to the simple SEIR model, in more complicated models, we still expect that the number of deaths averted by a lockdown imposed and lifted early in the epidemic will be $\mathrm{O}(\epsilon)$

While we have paid special attention to the question of fatalities averted, the argument above is general enough that it can be applied to any intrinsic property of the trajectory. For instance, one may introduce a compartment corresponding to hospitalized patients, and ask about the peak occupancy of this compartment. But the argument above tells us that, when parameterized by the susceptible population, the entire trajectory of the lockdown scenario is separated from the no-lockdown scenario by a difference proportional to $\left(\epsilon_{2}-\epsilon_{1}\right)$.

Therefore, the argument implies that the difference between this peak, in the lockdown and no-lockdown scenarios, will also be proportional to $\left(\epsilon_{2}-\epsilon_{1}\right)$. An early lockdown may delay the peak in terms of time, but it will not bring down its height.

In Appendix A we verify some of these predictions for one example of an extended SEIR model.

In this section, we have heuristically argued and verified that even in general compartmental models, the effect of a lockdown is small. The reader might correctly point out that if the population is large then even an $\mathrm{O}(\epsilon)$ fraction might translate into a large number, in terms of actual lives. However, it is important to understand that these $\mathrm{O}(\epsilon)$ predictions made by models are not reliable. As we explain in section 4 , the real-world impact of a lockdown is controlled by different factors, which are not accounted for in simple models, but can change the outcome of the epidemic much more significantly than these $\mathrm{O}(\epsilon)$ effects.

\section{Real-world caveats}

The results above apply in an idealized setting, and so we now describe several real-world caveats to the results. In reality, a lockdown may have both negative and positive impacts on the final toll of an epidemic. We list some of the factors that could lead to this. We are especially interested in those factors that might be relevant in India's case.

\subsection{Possible negative long-term impacts of a lockdown}

A lockdown may have a number of negative long-term impacts.

\section{Lockdown leading to larger long-term values of $\beta_{1}$}

We described above how the eventual toll of the epidemic is almost entirely controlled by its rate of spread after the lockdown ends; we denoted the relevant parameter by $\beta_{1}^{(a)}$ above. In reality, $\beta_{1}^{(a)}$ depends on long-term sustainable precautions that individuals can take. A lockdown that fails to emphasize welfare - as has been the case in India may make it harder for people to implement such precautions.

For instance, measures such as reducing the number of working days in a week, or implementing staggered hours for markets and shops to reduce crowding can contribute to a reduction of $\beta_{1}^{(a)}$. However, such precautions almost invariably have an economic cost. 
If the lockdown has engendered economic insecurity, and chipped away at the economic and social reserves of people, it may make it harder to implement such precautions sustainably in the long term.

Therefore, the lockdown, by itself, may cause an increase in the long term value of $\beta_{1}$, by making it harder for people to implement sustainable physical-distancing measures. This can easily overwhelm the very small $\mathrm{O}(\epsilon)$ direct gains in deaths averted due to the lockdown.

\section{Lockdown leading to a humanitarian crisis}

More than $90 \%$ of India's workforce is employed in the unorganized sector, or informally employed in the organized sector. Consequently, the economic impact of the lockdown has been severe. The Center for Monitoring Indian Economy estimated that, despite a slightly improvement in the month of May compared to the month of April, "over a 100 million people were still out of jobs compared to employment in 2019-20" [5]. Another glimpse of the extent of the crisis is provided by the observation made by the Stranded Workers Action Network (SWAN). SWAN contacted thousands of workers but found that "90\% ... did not get paid by their employers" and "96\% ... did not get rations from the government" [6].

This humanitarian crisis is a direct consequence of the lockdown, and constitutes the single most significant impact that the lockdown has had on India's people.

\section{Lockdown reducing access to healthcare}

A lockdown may make it harder for people to access healthcare for other conditions. It is known that tuberculosis (TB), by itself, claims about 450,000 lives every year in India. But, as a direct consequence of the lockdown, reports suggest that the number of new TB cases notified in government healthcare centers fell very sharply in April 2020 when compared with the previous year [7]. If the Indian lockdown causes the rate of TB deaths to rise by even a fraction, it could again easily overwhelm the $\mathrm{O}(\epsilon)$ gains made in combating COVID-19.

\subsection{Possible positive long-term impacts of a lockdown}

A lockdown may possibly have positive long-term effects although it is not clear if such effects are directly visible in India's case.

\section{Possibility of quashing the epidemic}

A lockdown may succeed in quashing the epidemic or reducing the epidemic to a very low level so that, through testing, tracing and other interventions, its rate of spread can subsequently be kept low for a long period. When the number of cases is very low, the dynamics of an epidemic cannot be reliably understood through compartmental models. For instance, at the time of writing, the lockdown in New Zealand is believed to have almost ended cases of COVID-19 there. Similarly, the lockdown in Hubei that provided 
the template for other lockdowns, brought the epidemic down to such a low level that the Chinese government has so far, through testing and tracing, succeeded in keeping the number of new infections very limited.

This is irrelevant for the discussion in India, where the epidemic has evidently not been quashed by the lockdown.

\section{Late lockdowns}

If a lockdown is imposed late in the epidemic, when the number of new infections is close to its peak, then even a simple lockdown may reduce the number of fatalities by reducing the "overshoot" beyond herd immunity. In this situation, the analysis of section 2 would not be valid since $\epsilon_{1}, \epsilon_{2}$ would not be small.

\section{Preparing the healthcare system}

A lockdown may reduce the absolute number of cases, and so reduce the immediate stress on the healthcare system. It may also provide time for the state to ramp up health facilities. We use this term to include the ramping-up of testing and quarantine facilities.

We note that the observation that the lockdown has currently prevented healthcare facilities from being overwhelmed does not, by itself, provide a compelling rationale for the lockdown. This involves the same logical error that we described above in the case of fatalities. If the lockdown merely delays the date on which the healthcare system is overwhelmed, then this implies that its long-term positive-effects are limited.

Therefore, the question that must be asked is whether the lockdown has been used to ramp up health facilities so that they are better-prepared to confront the epidemic in the long run.

\section{Behavioural changes}

It is sometimes argued that the lockdown was necessary to promote behavioural change and to persuade individuals to adopt precautions. We list this factor for completeness but we do not believe that this constitutes a genuinely long-term impact. For instance, while wearing masks in public or hand-washing where possible are both important precautions, it appears clear that the these precautions could have been encouraged even without the lockdown.

\section{A Vaccine}

If a vaccine for a disease is already available, the delay induced by a lockdown can, in principle, be used to vaccinate a large number of people. However, in the case of the COVID-19 pandemic, it appears clear that a vaccine will not be available at least for several months and perhaps for longer. Moreover, even after a safe vaccine becomes available, it will take a considerable amount of time to actually vaccinate a significant fraction of the world's population. Since these time-scales are so much longer than the 
time-scale of the delay induced by the Indian lockdown, the potential availability of a vaccine in the future cannot be used to evaluate the lockdown in India.

The objective of this note is not to enter into a detailed discussion of these qualitative effects. Here, we would merely like to point out that the factors above are far more important than meaningless measures of deaths-delayed by a fixed date. This is because even small changes in the factors above - either in the positive or the negative direction - can completely overwhelm the $\mathrm{O}(\epsilon)$ fatalities averted by the lockdown. So the lockdown must be gauged by evaluating whether the positive long-term effects above outweigh its negative long-term effects.

In principle, some of the effects above can be included as parameters in models. For instance one could include a "back-reaction effect" of the lockdown accounting for its impact on the long term value of $\beta_{1}$. But, the available data in India does not allow for a quantitative analysis of any of these factors, and we are not aware of any such analysis having been performed. So, at the moment, the comparison of the positive and negative effects of the lockdown must necessarily rely on a qualitative, and not a quantitative, analysis.

\section{Conclusion and discussion}

There is currently no available quantitative evidence that would suggest that the Indian lockdown has "averted" deaths, as opposed to "delaying" deaths.

Nevertheless, some groups have attempted to make such a claim by using SEIR-models or more-general compartmental models. These estimates appear to compare fatalities between scenarios by an arbitrarily chosen fixed date.

We showed that such claims are misleading and appear to arise from a lack of appreciation of some elementary aspects of the dynamics of such models. We emphasize that while our precise formulas were derived in the context of SEIR models in section 2, our heuristic arguments from section 3 are robust and also apply to more complicated models.

This means that question of whether the Indian lockdown has really averted any deaths must rely on a comparison of the effects described in section 4. A quantitative analysis of these effects is not possible given the extant data.

So the factors listed in section 4 must be compared qualitatively. In this note, we do not provide a detailed qualitative analysis of these factors. Nevertheless, to this author, it appears clear that the Indian lockdown has not led to any dramatic improvement in the ability of the healthcare system to address the COVID-19 pandemic. And the small steps that have been taken to ramp up the production of PPE, or increase testing appear to be far from sufficient to offset the humanitarian and healthcare crises caused by the lockdown, or even the negative impact that the lockdown has had on the ability of people to sustainably

maintain physical-distancing measures in the long run. Therefore, this author is of the opinion that the lockdown of the country has exacerbated the challenges of the COVID-19 pandemic and created the risk of excess deaths, rather than averting deaths. 
Viable alternatives to a nationwide lockdown, which were advocated by several public health experts, may have included early testing and tracing, localized lockdowns, and a promotion of more sustainable physical-distancing measures, together with a strong focus on the welfare of people affected by restrictions.

\section{Acknowledgments}

I am grateful to Pinaki Chaudhuri, Sourendu Gupta, Alok Laddha, Gautam Menon, Subroto Mukerjee, Madhusudhan Raman, Joseph Samuel, Srikanth Sastry, Ramachandran Shankar, Rahul Siddharthan and Supurna Sinha for comments on a draft of this note. I am also grateful to members of the Indian Scientists Response to COVID-19 collective and the Politically Mathematics collective for several discussions on related issues.

\section{Appendix}

\section{A The INDSCI-SIM model}

In this appendix, we discuss the INDSCI-SIM model [8], since this is an extended compartmental model and it was also used to generate one of the figures mentioned in the Introduction.

Although the predictions of INDSCI-SIM have been discussed in the popular media, as of the time of writing this note, the modellers had released neither a detailed preprint describing the model and related calculations, nor the computer code for the model. In the discussion below, we are forced to rely on an online tool that the modellers created, and some summary slides of documentation that they have put up. Both of these are accessible through the model's website. We found this information insufficient to recreate, in detail, the various steps that led to the final figure for "deaths averted" due to the lockdown that the modellers announced. Therefore we have been forced to make plausible inferences in what follows.

\section{Some generalities}

The INDSCI-SIM model differs from the SEIR model, since it has 9 compartments instead of 4, and additionally envisions a division of the Indian population into state-level populations that interact with each other. For instance, there are compartments corresponding to those who are "asymptomatic", "pre-symptomatic", "mildly symptomatic", "hospitalized" etc.

While the dynamics of this model are more complicated, it would be erroneous to conclude that they are a more accurate representation of reality as we now explain.

Even for a single sub-population, the model appears to have at least 14 parameters. Some of these are clinical: a parameter for the fraction of asymptomatic individuals, another parameter for the fraction for those who are pre-symptomatic but have mild symptoms etc. Some other parameters correspond to social dynamics: a parameter controlling the rate of contacts between asymptomatic and susceptible individuals, another controlling the same rate for mildly symptomatic individuals etc. If one consider multiple sub-populations, then there are additional parameters that control the contacts between these populations. 
What is common to all these parameters is that they are poorly understood and have certainly not been reliably quantified. As is well known to modellers, given a surfeit of parameters, it is possible to fit a variety of data. Moreover, by tweaking these parameters, it is often easy to obtain any result one wishes.

Nevertheless, as we explained above, the result that "a lockdown does not avert deaths, but only delays them," is rather robust. This result constitutes one of the few robust predictions made by models like INDSCI-SIM. The documentation for the model (slide 19 in v1.0) acknowledges this.

We used the model's online tool on 4 June 2020 to verify this property of the model. We maintained the tool's default settings, which seed the epidemic only in Maharashtra. We emphasize that these default parameters are not realistic. But, our point here is only to establish that even this model follows the robust predictions above.

We set the epidemic to start on 1 March, and allowed it to run for 300 days. We compared the following two scenarios. In the first scenario, no intervention was introduced. In the second scenario, we set the intervention to what the tool calls a "simple lockdown" beginning on day 20 and continuing till day 90 .

We found that, without the lockdown, the number of deaths in Maharashtra by 25 December was 31,372 . With the lockdown, the number of deaths by that date was 31,302 . So, in this example, the INDSCI-SIM model itself "predicts" that a simple lockdown of 70 days will save 70 lives asymptotically.

On the other hand, if one were to do something meaningless like comparing deaths by 15 May, then one would find in this example that, without the lockdown, there would be 8,400 deaths whereas the lockdown would bring this number down to 217 . But, of course, it would be extremely misleading to conclude that the lockdown has "averted more than 8,000 deaths". Even in this model, it has only delayed these deaths and they rapidly catch up with the no-lockdown scenario.

\section{Perspective on lockdowns}

In spite of this, the modellers suggest that a lockdown may be effective in the long term. (Slides 19 and 20 in v1.0.)

This is because, apart from the parameters above, the model introduces additional parameters corresponding to an exponentially increasing ability of the state to test and quarantine infected individuals. Mathematically, this is done by inserting an exponentially decaying intensity of contacts between infected and susceptible individuals. If $\gamma_{i}$ are the parameters that control this intensity (which are generalizations of the parameter $\beta_{1}$ in our discussion of the SEIR model), the INDSCI-SIM model sets

$$
\gamma_{i}(t)=\gamma_{i}(0) e^{-\frac{t}{\tau_{i}}}
$$

with different time-constants, $\tau_{i}$, for the different categories of infected individuals.

Since the parameters in the model now have an explicit time-dependence, the analysis of section 3.2 is no longer formally valid. In words, the assumption is that the period of the 
lockdown is used to exponentially improve the ability of the healthcare system to test and quarantine. And as we explained in section 4, it is true that, in principle, such an exponential improvement would lead to a long-term positive effect.

There are two important issues. The first is that the model does not include parameters for any of the negative long-term impacts of the lockdown, but only this possible positive impact. So the benefits of a lockdown are almost inbuilt into this model unless one sets $\tau_{i} \rightarrow \infty$.

But more seriously, there is no good independent measure of how much the healthcare system (including testing) has improved during the lockdown. As we see below, this turns out to be a genuine problem for this model since there is no good way to quantify the parameters $\tau_{i}$ that enter above.

\section{Prediction for deaths averted}

We now turn to the INDSCI-SIM claim that the Indian lockdown averted between 8,000 to 32,000 deaths and explain why this conclusion is baseless.

The recorded fatality data [4] for India is shown from 11 March (the data of the first fatality) to 15 May (the time frame for the analysis under consideration) in Figure 3.

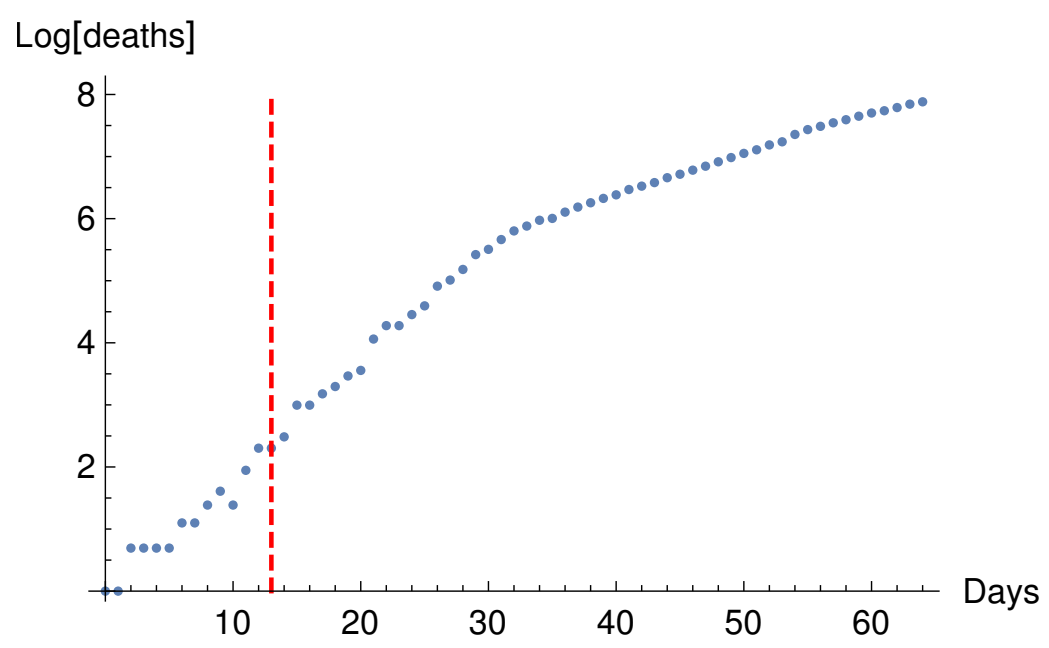

Figure 3: Logarithm of the number of recorded deaths in India from 11 March to 15 May. The start of the lockdown is marked with a dashed line.

The reader will immediately note that there are very few data points before the lockdown. In fact, the number of data points is smaller than the number of parameters for a single subpopulation in the INDSCI-SIM model.

Consequently, while the rate of growth of fatalities clearly slowed in April, there is no way to separate a number of effects above: the effect of the enforced physical distancing, the effect of increased testing-quarantining, the effect of behavioural changes and other precautions and the fact that the data might itself be flawed due to improvement in disease-surveillance over 
time [9]. But this division is extremely important for understanding whether the lockdown has had a long-term impact since, as we have explained in great detail above: (a) the effects of enforced physical distancing due to the lockdown are transient (b) the effects of behavioural changes are long-lasting but not necessarily related to the lockdown (c) the effects of increased testing-quarantining are long-lasting.

Faced with this difficulty, the modellers of INDSCI-SIM appear to have adopted the following procedure, as far as we can discern from v1.2 of the documentation. They simply estimated a rate of exponential growth by fitting the data until April 3. (The significance of this particular date is unclear to us.) Then they extrapolated this rate of exponential growth to May 15, and compared it with the known cases on that date. They declared that the difference of these two figures was the number of "deaths averted" by the lockdown. Since the estimate of the early exponential rate is prone to errors, which can have a significant impact over this time-period, the modellers obtained the large range of 8,000-32,000 "deaths averted."

We note two obvious points

1. The idea that the calculation above yields the "deaths averted" by the lockdown is absurd. In fact, if the slowdown in the rate of growth in Figure 3 arises from a combination of enforced physical distancing and behavioural changes (rather than increased testingquarantining), then our analysis shows that the final number of deaths averted by the lockdown itself - even before accounting for its other long-term negative impacts - is likely to be very small.

2. The simple analysis of extrapolating the rate of exponential growth beyond April 3 can be done without the INDSCI-SIM model, since any model would yield exponential growth in the early stages. Indeed, this is likely the same procedure that other groups mentioned in the Introduction used. Therefore, not only is the final figure mislead-

ing, it is also misleading to suggest that the computation has anything to do with a sophisticated epidemiological model.

\section{References}

[1] J. Jagannath, "Lockdown saved 37,000-78,000 lives, averted 14 lakh-29 lakh Covid-19 cases: Govt," Mint, 22 May 2020. Available from: https://www.livemint.com/news/india/lockdown-saved-37-000-78-000-lives-averted14-lakh-29-lakh-covid-19-cases-govt-11590147462140.html [accessed 13 June 2020].

[2] Pinaki Chaudhuri and Gautam Menon, "Explained: How many Covid-19 deaths prevented by lockdown?," Indian Express, 4 June 2020. Available from: https://indianexpress. com/article/explained/how-many-deaths-prevented-6441365/ [accessed 13 June 2020].

[3] L. J. Allen, F. Brauer, P. Van den Driessche, and J. Wu, Mathematical epidemiology, vol. 1945. Springer, 2008. 
[4] CSEE at Johns Hopkins University. "CSSEGISandData," [online]. 2020 [accessed 16 June 2020]. Available from: https://github.com/CSSEGISandData.

[5] Mahesh Vyas. "21 million jobs added in May," [online]. 2 June 2020 [accessed 17 June 2020]. Available from: https://www. cmie.com/kommon/bin/sr.php?kall=warticle\&dt=2020-06$02 \% 2011: 43: 41 \mathrm{\& msec}=800$.

[6] Data Team, "Data - 96\% migrant workers did not get rations from the government, $90 \%$ did not receive wages during lockdown: survey," The Hindu, 20 April 2020. Available from: https://www.thehindu.com/data/data-96-migrant-workers-did-not-get-rations-fromthe-government-90-did-not-receive-wages-during-lockdown-survey/article31384413. ece [accessed 13 June 2020].

[7] Aneesha Bedi and Swagata Yadavar, "TB patients badly hit by lockdown $80 \%$ drop in diagnosis, huge struggle for medicines," The Print, 30 April 2020. Available from: https://theprint.in/health/tb-patients-badly-hit-by-lockdown-80-drop-indiagnosis-huge-struggle-for-medicines/411399/ [accessed 13 June 2020].

[8] Snehal Shekatkar, Bhalchandra Pujari, Mihir Arjunwadkar, Dhiraj Kumar Hazra, Pinaki Chaudhuri, Sitabhra Sinha, Gautam I Menon, Anupama Sharma, and Vishwesha Guttal, "INDSCI-SIM A state-level epidemiological model for India," 2020. Available from: https://indscicov.in/indscisim [accessed 13 June 2020].

[9] Sourendu Gupta, "Epidemic parameters for covid-19 in several regions of india," 2020. arXiv:2005.08499. Available from: http://www.arxiv.org/abs/2005.08499. 